\title{
Duality relation between nonspherical mirror optical cavities and its application to gravitational-wave detectors
}

\author{
Juri Agresti, ${ }^{1,2, *}$ Yanbei Chen, ${ }^{3,4}$ Erika D'Ambrosio, ${ }^{2}$ and Pavlin Savov ${ }^{3}$ \\ ${ }^{1}$ Istituto di Fisica Applicata "Nello Carrara"-CNR, 50019 Sesto Fiorentino (FI), Italy \\ ${ }^{2}$ LIGO Laboratory, California Institute of Technology, Pasadena, California 91125, USA \\ ${ }^{3}$ Theoretical Astrophysics, California Institute of Technology, Pasadena, California 91125, USA \\ ${ }^{4}$ Max-Planck-Institut für Gravitationsphysik (Albert-Einstein-Institut), D-14476 Golm, Germany \\ ${ }^{*}$ Corresponding author: j.agresti@ifac.cnr.it
}

Received May 24, 2012; revised July 6, 2012; accepted July 9, 2012;

posted July 10, 2012 (Doc. ID 168898); published August 9, 2012

\begin{abstract}
In this paper, we analytically prove a unique duality relation between the eigenspectra of paraxial optical cavities with nonspherical mirrors: a one-to-one mapping between eigenmodes and eigenvalues of cavities deviating from flat mirrors by $h(\vec{r})$ and cavities deviating from concentric mirrors by $-h(\vec{r})$, where $h$ need not be a small perturbation. We then illustrate its application to optical cavities, proposed for advanced interferometric gravitationalwave detectors, where the mirrors are designed to support beams with rather flat intensity profiles over the mirror surfaces. This unique mapping might be very useful in future studies of alternative optical designs for advanced gravitational wave interferometers or experiments employing optical cavities with nonstandard mirrors. @ 2012 Optical Society of America

OCIS codes: $\quad 070.2580,080.4228,260.0260,230.0230,140.0140$.
\end{abstract}

\section{INTRODUCTION}

Laser Interferometer Gravitational-Wave Observatory (LIGO) [1] and other long baseline detectors are formed by highfinesse Fabry-Perot arms in order to increase the circulating optical power and to enhance sensitivities by suppressing shot noise. LIGO interferometers, as well as the baseline design for advanced-LIGO detectors [2], all use spherical mirrors and fundamental Gaussian mode. Mirror thermal noise is expected to be the dominant source of noise in the most sensitive frequency band of second-generation, ground-based gravitational-wave detectors. Different shapes of beam have been proposed for reducing this noise, such as rather flat mesa-like [3] mode called Mesa beams [4-8], conical modes [9], and high-order Laguerre-Gauss modes $[\underline{10}, \underline{11}]$. In particular, the former two approaches and more general optimized beam profiles [12] require the use of nonspherical mirrors in the Fabry-Perot optical cavities. The research on Mesa beams and nonspherical mirrors supporting them was very active in the past few years and led to the discovery of the duality relation as described in the following.

Ambrosio et al. []ㅡ, O'Shaughnessy et al. []], and Agresti and DeSalvo [13] calculated that the thermal fluctuations of mirror surfaces are better averaged by Mesa beams with respect to Gaussian ones. The corresponding optical design has shown a strong tilt instability [14], and Thorne has proposed a different version of the Mesa beam, which is supported by nearly concentric and opportunely shaped mirrors; this new version provides the same intensity profile at the cavity mirrors (and thus the same thermal noise), but implies a weaker tilt instability (even weaker than cavities with nearly concentric spherical mirrors analyzed by Sigg and Sidles
$[15,16])$ - as calculated by Savov and Vyatchanin [14]. A general method to design a family of optical cavities from nearly flat to nearly concentric ones has been devised by Bondarescu and Thorne [17], and the resulting fundamental mode, called a hyperboloidal beam, was later studied in more detail as an alternative to Mesa beams $[\underline{18}, \underline{19}]$.

Mesa beams are constructed by coherently overlapping Gaussian beams, with either (i) translated parallel axes, or (ii) axes in different directions but sharing a common midpoint [17]. Mirror shapes that support such beams as fundamental modes are derived from the phase fronts at the mirror locations, with case (i) corresponding to Mexican-hat mirrors and case (ii) corresponding to the nearly concentric version. Using the resulting optics profile, higher-order optical modes and eigenfrequencies of the designed cavities must be calculated by solving an eigenvalue problem, which has been done for nearly flat cavities by D'Ambrosio et al. [5] and O'Shaughnessy et al. [6], and for nearly concentric cavities by Savov and Vyatchanin [14]. During his numerical work, Savov discovered that the deviation of nearly concentric Mexican-hat mirrors from concentric surfaces is exactly the opposite of the deviation of nearly flat Mexican-hat mirrors from flat surfaces; he also found that the corresponding higher modes of these cavities all have the same intensity profiles, and that there is a one-to-one mapping between their eigenvalues. Following this numerical analysis, Bondarescu conjectured a general duality relation between axisymmetric cavities with two identical mirrors facing each other: cavities with mirrors deviating by $-h(|\vec{r}|)$ from concentric surfaces (nearly concentric mirrors) will support modes with the same intensity profiles and related eigenvalues as cavities with mirrors deviating by $h(|\vec{r}|)$ from flat surfaces (nearly flat 
mirrors). It should be noted that the deviation $h(\vec{r})$ is not required to be infinitesimal; it can change the mirror shape arbitrarily as long as the paraxial approximation is still satisfied. Here and henceforth in the paper a two-dimensional (2D) vector $\vec{r}$ has been used to indicate each point on planes orthogonal to the cavity axis. While such a duality relation is well-known between cavities with spherical mirrors, i.e., those with $h(\vec{r}) \sim \alpha \vec{r}^{2}$ (for example, see [20-24]), to our best knowledge no such relations had been established between generic cavities.

In this paper, we prove this remarkable correspondence analytically, for an even broader category of cavities: those whose mirror shapes remain invariant under the parity operation, identified as spatial reflection in the $2 \mathrm{D} \vec{r}$-space (which is also equivalent to a $180^{\circ}$ rotation around the cavity axis). Eigenmodes of such cavities can be put into eigenstates of parity, and we show that all corresponding eigenmodes of dual cavities have the same intensity profiles at the mirrors, with their eigenvalues satisfying

$$
\gamma_{\mathrm{c}}^{k}=(-1)^{p_{k}+1} e^{-2 i k L}\left(\gamma_{\mathrm{f}}^{k}\right)^{*}
$$

where $(-1)^{p_{k}}$ is the parity of the $k$ th eigenmode; subscripts $c$ and $\mathrm{f}$ denote nearly concentric and nearly flat mirrors, respectively.

We will give two alternative proofs of this duality relation. The first one relies on the geometrical properties of the propagator from mirror to mirror. In this description, the eigenfunctions are field amplitudes at mirror surfaces, and we see right away that the corresponding eigenstates have the same intensity profiles there. The second proof is based on the "center-to-center" propagator. The center-of-the-cavity fields are the eigenstates and the correspondence relation is manifested by a 2D Fourier transform, which univocally relates the dual cavities.

This paper is organized as follows. In Section $\underline{2}$ we report the first proof; in Subsection 2.A, the Cartesian coordinates are used and some general features of the eigenproblem are described, and in Subsection 2.B, the cylindrical coordinates are used, and the case of axisymmetric resonators is studied. Section 3 contains the second proof and the 2D Fouriertransform relation between the center-of-the-cavity eigenmodes of dual cavities. Section 4 specializes to the case of Mexican-hat cavities. When the nearly flat and the nearly concentric mirrors are implemented in the system, the corresponding Mesa beams are connected by Fourier transform, as we report in Subsection 4.A. In Subsection 4.B, plots and analytical forms are provided, for the amplitude distributions at the center of the cavity and at the mirror surfaces; in Subsection 4.C, we address the tilt instability of the nearly concentric Mexican-hat resonator and show how easily it can be analyzed, applying the duality relation to the results obtained for the nearly flat Mexican-hat cavities [5,6]. We comment and review the implications of the general duality in Section 5 .

\section{ANALYTICAL PROOF FOR MIRROR-TO- MIRROR PROPAGATION}

\section{A. In the Cartesian Coordinate System}

In this section we focus on field distributions on mirror surfaces, and restrict ourselves to cavities with two identical mirrors facing each other. The extension to asymmetric cavities is presented in Appendix A. We adopt the FresnelKirchoff diffraction formula to propagate fields from mirror surface to mirror surface (see, e.g., [20]). In this formalism, the field amplitude $v_{1}\left(\vec{r}^{\prime}\right)$ on the surface of mirror 1 propagates into

$$
v_{2}(\vec{r})=\int \mathrm{d}^{2} \vec{r}^{\prime} \mathcal{K}\left(\vec{r}, \vec{r}^{\prime}\right) v_{1}\left(\vec{r}^{\prime}\right)
$$

on mirror 2 , via the propagator

$$
\mathcal{K}\left(\vec{r}, \vec{r}^{\prime}\right)=\frac{i k}{4 \pi \rho}(1+\cos \theta) e^{-i k \rho} \quad k=\frac{2 \pi}{\lambda},
$$

from $\vec{r}^{\prime}$ (on mirror 1) to $\vec{r}^{\prime}$ (on mirror 2), where $\rho$ denotes the [(three-dimensional (3D)] spatial distance between these two points and $\theta$ stands for the angle between the cavity axis and the reference straight line, as is illustrated in Fig. 1. We know that the Fresnel-Kirchoff integral eigenequation

$$
\gamma v(\vec{r})=\int \mathrm{d}^{2} \vec{r} \mathcal{K}\left(\vec{r}, \vec{r}^{\prime}\right) v\left(\vec{r}^{\prime}\right)
$$

univocally determines the eigenmodes $v$ and eigenvalues $\gamma$ of the cavity.

Applying the paraxial approximation

$$
\theta \approx 0, \quad \rho \approx L+\frac{\left|\vec{r}-\vec{r}^{\prime}\right|^{2}}{2 L}-h(\vec{r})-h\left(\vec{r}^{\prime}\right),
$$

we can use

$$
\mathcal{K}_{\mathrm{f}}^{h}\left(\vec{r}, \vec{r}^{\prime}\right)=\frac{i k}{2 \pi L} e^{-i k L} e^{i k h(\vec{r})} e^{-\frac{i k}{2 L}\left|\vec{r}-\vec{r}^{\prime}\right|^{2}} e^{i k h\left(\vec{r}^{\prime}\right)}
$$

in the integral eigenequation.

Here the mirror surfaces deviate by $h(\vec{r})$ from a flat reference, and the subscript $f$ is used to reflect this convention. From here on, we will also refer to $\mathcal{K}_{\mathrm{f}}^{h}$ as the nearly flat propagator. We now consider two slightly deformed concentric mirrors (see Fig. 2) so that the mirror's height with respect to the flat reference surface is

$$
h(\vec{r})=\vec{r}^{2} / L+b(\vec{r}),
$$

where the height $b(\vec{r})$ is the deviation from the concentric spherical surface (note that concentric spherical mirrors have

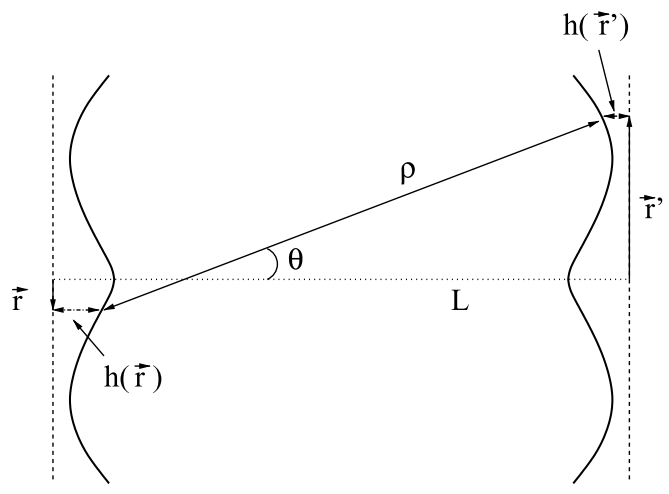

Fig. 1. Symmetric nearly flat mirrors. 


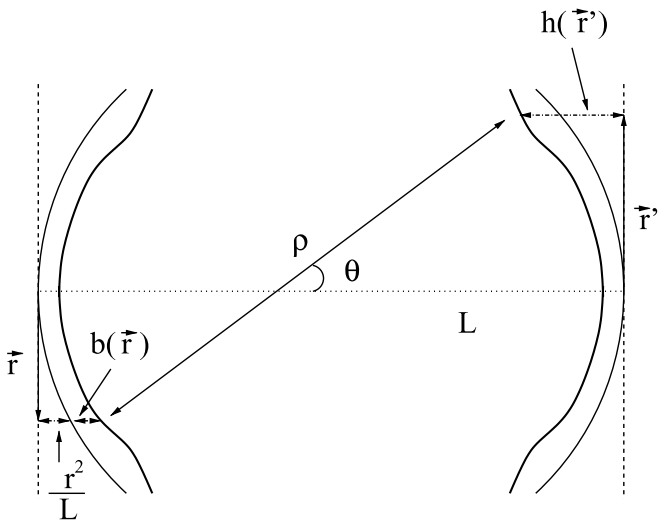

Fig. 2. Symmetric nearly concentric mirrors.

their radii of curvature equal to $L / 2$, and thus surface height $\left.r^{2} / L\right)$. Inserting Eq. (2.6) into Eq. (2.4), we obtain the propagator for a nearly concentric cavity,

$$
\mathcal{K}_{c}^{b}\left(\vec{r}, \vec{r}^{\prime}\right)=\frac{i k}{2 \pi L} e^{-i k L} e^{i k b(\vec{r})} e^{+\frac{i k}{2 L}\left|\vec{r}+\vec{r}^{\prime}\right|^{2}} e^{i k b\left(\vec{r}^{\prime}\right)}
$$

We use the term nearly concentric propagator for $\mathcal{K}_{c}^{b}\left(\vec{r}, \vec{r}^{\prime}\right)$. Although we use the terms nearly flat and nearly concentric, $h$ and $b$ are not required to be small; in fact, they can represent any deviation from perfectly flat and concentric spherical mirrors.

Now let us consider mirrors that are then invariant under parity, i.e., those in which we also have

$$
h(\vec{r})=h(-\vec{r}), \quad b(\vec{r})=b(-\vec{r})
$$

so that $\mathcal{K}_{f, c}$ are both invariant under a spatial reflection

$$
\left\{\vec{r}, \vec{r}^{\prime}\right\} \leftrightarrow\left\{-\vec{r},-\vec{r}^{\prime}\right\}
$$

and, therefore, we have

$$
\mathcal{P K}=\mathcal{K} \mathcal{P}
$$

where we have defined

$$
\mathcal{P} v(\vec{r})=v(-\vec{r})
$$

for $2 \mathrm{D}$ reflection. Equation (2.10) implies that all eigenmodes can be put into forms with definite parity. We derive the following relation between nearly flat and nearly concentric propagators, as constructed:

$$
\left[\mathcal{K}_{\mathrm{f}}^{h}\left(-\vec{r}, \vec{r}^{\prime}\right)\right]^{*}=-e^{2 i k L} \mathcal{K}_{c}^{-h}\left(\vec{r}, \vec{r}^{\prime}\right)
$$

which is equivalent to

$$
\mathcal{P}\left[\mathcal{K}_{\mathrm{f}}^{h}\right]^{*}=-e^{2 i k L} \mathcal{K}_{\mathrm{c}}^{-h}
$$

Suppose we have an eigenstate $v_{\mathrm{f}}$ of $\mathcal{K}_{\mathrm{f}}^{h}$, i.e., an eigenstate of a cavity with mirror deviating by $(+h)$ from flat surface, and we compute its eigenvalue $\gamma_{\mathrm{f}}$ and know the parity eigenvalue $(-1)^{p}$ :

$$
\begin{aligned}
\mathcal{K}_{\mathrm{f}}^{h} v_{\mathrm{f}} & =\gamma_{\mathrm{f}} v_{\mathrm{f}}, \\
\mathcal{P} v_{\mathrm{f}} & =(-1)^{p} v_{\mathrm{f}} .
\end{aligned}
$$

By applying Eqs. (2.13)-(2.15), we derive the correspondence

$$
\mathcal{K}_{\mathrm{c}}^{-h} v_{\mathrm{f}}^{*}=e^{-2 i k L}(-1)^{p+1} \gamma_{\mathrm{f}}^{*} v_{\mathrm{f}}^{*},
$$

which identifies $v_{\mathrm{c}} \equiv v_{\mathrm{f}}^{*}$ as the corresponding eigenstate of $\mathcal{K}_{c}^{-h}$, which is the eigenstate of the corresponding resonator we denote the dual. The eigenvalue is $\gamma_{\mathrm{c}} \equiv e^{-2 i k L}(-1)^{p+1} \gamma_{\mathrm{f}}^{*}$. We also induce that the parity is still $(-1)^{p}$. The reverse is straightforward and the result is an established one-to-one correspondence between dual cavities. We summarize this mapping in Table 1 . It is obvious to note that the corresponding eigenstates, $v_{\mathrm{f}}$ and $v_{\mathrm{f}}^{*}$, have the same intensity profiles on the mirror surfaces; for infinite mirrors, we know $v_{\mathrm{f}}(\vec{r})$ is real-valued (see Appendix B), so it is an eigenstate of the dual configuration itself.

For cavities with identical mirrors facing each other, the full, round-trip propagator is just the square of the half-trip one. From Eqs. (2.12) and (2.10), we have

$$
\left[\left[\mathcal{K}_{\mathrm{f}}^{h}\right]^{2}\right]^{*}=e^{4 i k L}\left[\mathcal{K}_{\mathrm{c}}^{-h}\right]^{2},
$$

which means that the same duality correspondence exists between eigenstates of the full propagator, with their eigenvalues related by

$$
\eta_{\mathrm{c}}=e^{-4 i k L} \eta_{\mathrm{f}}^{*}
$$

Note that when $h(\vec{r})=r^{2} /(2 L)$, the two dual cavities are identical to each other. Using the relation that links the eigenvalues of two dual resonators, we can determine the spectrum

$$
\gamma_{c}= \pm e^{-2 i k L} \gamma_{f}^{*}=\gamma_{f}=e^{-i k L+i n \pi / 2},
$$

where $n \in \mathcal{N}$. The resulting separation between the eigenvalues is the Gouy phase

$$
e^{i \theta_{G}}=e^{i \arccos (1-L / R)} \quad R=L
$$

computed for confocal resonators [20,22,24].

\section{B. Specializing to Cylindrical Mirrors}

In most practical applications cavity mirrors have cylindrical shapes: $h(\vec{r})=h(|\vec{r}|)$. This allows us to decouple radial and azimuthal degrees of freedom, and simplify the eigenvalue problem. We shall follow roughly the notation of [22].

Table 1. Correspondence of Propagation Kernels, Eigenstates, Parities, and Eigenvalues Between Dual Configurations

\begin{tabular}{lcc}
\hline & Nearly flat & Nearly concentric \\
\hline Kernel & $\mathcal{K}_{\mathrm{f}}^{h}$ & $\mathcal{K}_{\mathrm{c}}^{-h}$ \\
Eigenstate & $v_{\mathrm{f}}$ & $v_{\mathrm{f}}^{*}$ \\
Parity & $(-1)^{p}$ & $(-1)^{p}$ \\
Half-trip eigenvalue & $\gamma_{\mathrm{f}}$ & $e^{-2 i k L}(-1)^{p+1} \gamma_{\mathrm{f}}^{*}$ \\
Round-trip eigenvalue & $\eta_{\mathrm{f}}$ & $e^{-4 i k L} \eta_{\mathrm{f}}^{*}$ \\
\hline
\end{tabular}


We adopt the cylindrical coordinate system:

$$
\vec{r}=r(\cos \varphi, \sin \varphi) .
$$

Since $\mathcal{K}$ is now invariant under rotation along the $z$-axis, all eigenmodes can be put into eigenstates of rotation:

$$
v(r, \varphi)=R(r) e^{-i m \varphi}, \quad m=\text { integer }
$$

Inserting this into the eigenequation (2.3) and performing analytically the angular integration, we obtain the radial eigenequation

$$
\gamma_{n m} R_{n m}(r)=\int_{0}^{a} K_{\mathrm{f}(m)}^{h}\left(r, r^{\prime}\right) R_{n m}\left(r^{\prime}\right) r^{\prime} \mathrm{d} r^{\prime}
$$

where for each angular mode number $m$ we have indexed the radial eigenstates by $n$, and

$$
K_{\mathrm{f}(m)}^{h}\left(r, r^{\prime}\right)=\frac{i^{m+1} k}{L} J_{m}\left(\frac{k r r^{\prime}}{L}\right) e^{i k\left[-L+h(r)+h\left(r^{\prime}\right)-\frac{r^{2}+r^{2}}{2 L}\right]}
$$

is a symmetric radial propagator, in the nearly flat description. Here we have used $J_{n}(z)=1 /\left(2 \pi i^{n}\right) \int_{0}^{2 \pi} e^{i z \cos \varphi} e^{i n \varphi} \mathrm{d} \varphi$, where $J_{n}(z)$ is the $n$ th-order Bessel function of the first kind. Since $K_{\mathrm{f}(m)}^{h}\left(r, r^{\prime}\right)$ is symmetric, we obtain orthogonality relations between radial eigenfunctions:

$$
\int_{0}^{a} R_{n_{1} m}(r) R_{n_{2} m}(r) r \mathrm{~d} r=\delta_{n_{1} n_{2}} .
$$

Using Eq. (2.6) again, for a configuration with $b(r)$ correction from concentric spherical mirrors, we obtain the radial kernel of the nearly concentric description:

$$
K_{\mathrm{c}(m)}^{b}\left(r, r^{\prime}\right)=\frac{i^{m+1} k}{L} J_{m}\left(\frac{k r r^{\prime}}{L}\right) e^{i k\left[-L+b(r)+b\left(r^{\prime}\right)+\frac{r^{2}+r^{\prime 2}}{2 L}\right]} .
$$

Comparing Eqs. (2.24) and (2.22), we obtain

$$
(-1)^{m+1}\left[K_{\mathbf{f}(m)}^{h}\right]^{*}=e^{2 i k L} K_{\mathrm{c}(m)}^{-h} .
$$

This is a radial version of Eq. (2.13); here we know explicitly that all $m$-eigenstates have parity $(-1)^{m}$.

Following a similar reasoning as done in the previous section, for each angular mode number $m$, we can establish a one-to-one correspondence between radial eigenstates of a nearly flat configuration and those of the dual configuration:

$$
\left[R_{n m}\right]_{\mathrm{c}}=\left[R_{n m}\right]_{\mathrm{f}}^{*} .
$$

The mapping of the eigenvalues is given by

$$
\left[\gamma_{n m}\right]_{\mathrm{c}}=(-1)^{m+1} e^{-2 i k L}\left[\gamma_{n m}\right]_{\mathrm{f}}^{*} .
$$

Similarly, the round-trip eigenstates have the same correspondence, their eigenvalues related by

$$
\left[\eta_{n m}\right]_{\mathrm{c}}=e^{-4 i k L}\left[\eta_{n m}\right]_{\mathrm{f}}^{*} .
$$

\section{ANALYTICAL PROOF BASED ON CENTER-TO-CENTER PROPAGATION}

A. Propagators for Vacuum and Mirror Surfaces

In this subsection, we focus on complex amplitudes of the optical field on planes perpendicular to the optical axis (the $z$-axis). An optical mode propagating along one direction of the optical axis can be specified completely by the distribution of the field on the $z=$ const plane. For example, we denote the optical field on the plane $z=z_{1}$ by $v\left(\vec{r}, z_{1}\right)$, where $\vec{r}$ is the 2D coordinate of the point on this plane. The effect of any linear paraxial optical system (including open space, thin lenses, and mirrors) with input plane $z_{1}$ and output plane $z_{2}$ can be characterized by its transfer operator, $\mathcal{U}$, which takes the form of an integration kernel:

$$
v\left(\vec{r}, z_{2}\right)=\int \mathrm{d}^{2} \vec{r}^{\prime} \mathcal{U}\left(\vec{r}, z_{2} ; \vec{r}^{\prime}, z_{1}\right) v\left(\vec{r}^{\prime}, z_{1}\right)
$$

In particular, the operator that describes the paraxial propagation down a length $L$ in vacuum is

$$
\mathcal{G}_{L}\left(\vec{r}, \vec{r}^{\prime}\right)=i \frac{k}{2 \pi L} e^{-i k L} \exp \left[-i k \frac{\left(\vec{r}-\vec{r}^{\prime}\right)^{2}}{2 L}\right]
$$

For a mode propagating in the $\pm z$ direction with field (complex) amplitude distribution $v\left(\vec{r}^{\prime}, z_{1}\right)$ at $z=z_{1}$, the amplitude distribution on a surface described by height $z(\vec{r})=z_{1} \mp h(\vec{r})$ is given by

$$
v[\vec{r}, z(\vec{r})]=e^{ \pm i k h(\vec{r})} v\left[\vec{r}, z_{1}\right]
$$

Here we emphasize that the spatial point of interest is located outside the $z=z_{1}$ plane, and that the $2 \mathrm{D}$ vector $\vec{r}$ describes the projection of that point onto the $z=z_{1}$ plane.

From Eq. (3.3), one deduces that the operator for reflection off a perfect infinite mirror with shape $h(\vec{r})$ is

$$
\mathcal{R}_{[h(\vec{r})]}\left(\vec{r}, \vec{r}^{\prime}\right) \equiv-\delta\left(\vec{r}-\vec{r}^{\prime}\right) e^{2 i k h(\vec{r})} .
$$

The minus sign in Eq. (3.4) is used because we use a convention in which a phase shift by $\pi$ is gained upon reflection. It is easy to verify that both $\mathcal{G}_{L}$ and $\mathcal{R}_{[h(\vec{r})]}$ are unitary operators.

\section{B. Analytical Proof Based on Center-to-Center Propagation}

In this subsection we present an alternative proof motivated from the construction of the Mesa beams [6,14]: (i) the nearly flat configuration has its fundamental mode generated by spatial translation of minimal Gaussian beams, while (ii) the nearly concentric configuration is generated by rotation (of propagation direction at the center of cavity) of minimal Gaussian beams, or a translation in the momentum $k$-space. This had led us to speculate that the two sets of eigenstates correspond to each other via Fourier transform (similar to the relation between position and momentum space in quantum mechanics).

We will use the operator $\mathcal{G}_{L / 2}$ [see Eq. (3.2)], which propagates the field forward by half the cavity length. For simplicity, we denote it by $\mathcal{G}$ : 


$$
\mathcal{G}\left(\vec{r}, \vec{r}^{\prime}\right) \equiv i \frac{k}{\pi L} e^{-i k L / 2} e^{-i k \frac{\left(\vec{r}-\vec{r}^{\prime}\right)^{2}}{L}}
$$

Using $\mathcal{G}$ and $\mathcal{R}_{h(\vec{r})}$ [defined in Eq. (3.4), with $h(\vec{r})$ the mirror surface height], we can reexpress the eigenvalue problem as

$$
\mathcal{L}_{[h(\vec{r})]} u \equiv \mathcal{G} \mathcal{R}_{[h(\vec{r})]} \mathcal{G} u=\gamma u,
$$

with $\mathcal{L}_{[h(\vec{r})]}$ the center-to-center propagator when the mirror deviates from flat surfaces by $h(\vec{r})$, in which the optical mode propagates from the cavity center to the mirror, gets reflected, and propagates back to the center. In fact, $\mathcal{L}$ is related to the surface-to-surface propagator $\mathcal{K}$ by a unitary transformation,

$$
\mathcal{L}=\mathcal{G}^{-1} \mathcal{R}_{[h(\vec{r}) / 2]}^{-1} \mathcal{K} \mathcal{R}_{[h(\vec{r}) / 2]} \mathcal{G}
$$

This means the two proofs are mathematically equivalent. Similar to $\mathcal{K}$, the operator $\mathcal{L}$ also commutes with parity, or [cf. Eq. (2.10)]

$$
\mathcal{P} \mathcal{L}=\mathcal{L} \mathcal{P}
$$

With the propagator on hand, we proceed with our intuition that the modes must be related by Fourier transforms. In order to do so, we first define the 2D Fourier-transform operator $\mathcal{F}$ as

$$
\mathcal{F}\left(\vec{r}, \vec{r}^{\prime}\right)=\frac{k}{\pi L} e^{-\frac{2 i k}{L} \cdot \vec{r} \cdot \vec{r}^{\prime}},
$$

which satisfies

$$
\mathcal{F}^{2}=\left(\mathcal{F}^{-1}\right)^{2}=\mathcal{P}
$$

It is easy to show that

$$
\begin{aligned}
& {\left[\mathcal{G}^{*} \mathcal{F}^{-1}\right]\left(\vec{r}, \vec{r}^{\prime}\right)=-\frac{i k^{2}}{\pi^{2} L^{2}} e^{i k\left[\frac{L}{2}+\frac{\vec{r}^{2}}{L}+\frac{\left(\vec{r}-\vec{r}^{\prime}\right)^{2}}{L}\right]} \int \mathrm{d}^{2} \vec{r}^{\prime \prime} e^{\left.\frac{i k}{L} \vec{r}{ }^{\prime \prime}-\left(\vec{r}-\vec{r}^{\prime}\right)\right]^{2}}} \\
& =\left[i e^{i k L} \mathcal{R}_{\left[\vec{r}^{2} /(2 L)\right]} \mathcal{G}\right]\left(\vec{r}, \vec{r}^{\prime}\right) .
\end{aligned}
$$

[The integral on the second line can be done by inserting a factor $e^{-\epsilon\left(\vec{r}^{\prime \prime}\right)^{2}}$ into the integrand, and then letting $\epsilon \rightarrow 0^{+}$.] Similarly, [or by taking the transpose of Eq. (3.11)], we have

$$
\mathcal{F}^{-1} \mathcal{G}^{*} i e^{i k L} \mathcal{G R}_{\left[\vec{r}^{2} /(2 L)\right]} .
$$

Using Eqs. (‥11) and (‥12), we have

$$
\begin{aligned}
\mathcal{P} \mathcal{L}_{\left[h_{\mathrm{A}}\right]}^{*} & =\mathcal{F}^{-1}\left(\mathcal{F}^{-1} \mathcal{G}^{*}\right) \mathcal{R}_{\left[-h_{\mathrm{A}}\right]}\left(\mathcal{G}^{*} \mathcal{F}^{-1}\right) \mathcal{F} \\
& =-e^{2 i k L} \mathcal{F}^{-1} \mathcal{G} \mathcal{R}_{\left[\vec{r}^{2} /(2 L)\right]} \mathcal{R}_{\left[-h_{\mathrm{A}}\right]} \mathcal{R}_{\left[\vec{r}^{2} /(2 L)\right]} \mathcal{G} \mathcal{F} \\
& =-e^{2 i k L} \mathcal{F}^{-1} \mathcal{L}_{\left[h_{\mathrm{B}}\right]} \mathcal{F} .
\end{aligned}
$$

Here $h_{\mathrm{A}}$ and $h_{\mathrm{B}}$ are mirror heights related by the duality relation,

$$
h_{A}(\vec{r})+h_{B}(\vec{r})=r^{2} / L,
$$

and we have used the fact that

$$
\mathcal{R}_{\left[\vec{r}^{2} /(2 L)\right]} \mathcal{R}_{\left[-h_{\mathrm{A}}\right]} \mathcal{R}_{\left[\vec{r}^{2} /(2 L)\right]}=\mathcal{R}_{\left[\vec{r}^{2} / L-h_{A}\right]}=\mathcal{R}_{\left[h_{\mathrm{B}}\right]}
$$

According to Eq. (3.13), given any eigenstate $u_{A}$ of $\mathcal{L}_{\left[h_{\mathrm{A}}\right]}$ with eigenvalue $\gamma_{\mathrm{A}}$ and a definite parity of $p$, we have

$$
\begin{gathered}
(-1)^{p} \gamma_{\mathrm{A}}^{*} u_{A}^{*}=\mathcal{P} \mathcal{L}_{\left[h_{\mathrm{A}}\right]}^{*} u_{A}^{*}=-e^{2 i k L} \mathcal{F}^{-1} \mathcal{L}_{\left[h_{\mathrm{B}}\right]}\left(\mathcal{F} u_{A}^{*}\right), \\
\Rightarrow \mathcal{L}_{\left[h_{\mathrm{B}}\right]}\left(\mathcal{F} u_{A}^{*}\right)=(-1)^{p+1} e^{-2 i k L} \gamma_{\mathrm{A}}^{*}\left(\mathcal{F} u_{A}^{*}\right) .
\end{gathered}
$$

In other words, the mapping

$$
u_{\mathrm{A}} \rightarrow u_{\mathrm{B}}=\mathcal{F} u_{A}^{*}
$$

transforms each eigenstate of $\mathcal{L}_{\left[h_{\mathrm{A}}\right]}$ into its dual one of $\mathcal{L}_{\left[h_{\mathrm{B}}\right]}$; the corresponding eigenvalue relation is

$$
\gamma_{\mathrm{B}}=(-1)^{p+1} e^{-2 i k L} \gamma_{\mathrm{A}}^{*}
$$

For similar reasons, given any eigenstate $u_{\mathrm{B}}$ of $U_{\left[h_{\mathrm{B}}\right]}$ (with definite parity), $\mathcal{F} u_{\mathrm{B}}^{*}$ must also be an eigenstate of $U_{\left[h_{\mathrm{A}}\right]}$. Moreover, since

$$
\mathcal{F}\left(\mathcal{F} u_{\mathrm{B}}^{*}\right)^{*}=\mathcal{F} \mathcal{F}^{-1} u_{\mathrm{B}}=u_{\mathrm{B}}
$$

the state $\mathcal{F} u_{\mathrm{B}}^{*}$ is in fact the inverse image of $u_{\mathrm{B}}$ [under the mapping (3.18)]. This means we have established a one-toone correspondence between eigenstates of $\mathcal{L}_{\left[h_{\mathrm{A}}\right]}$ and those of $\mathcal{L}_{\left[h_{\mathrm{B}}\right]}$.

Now let us look at intensity profiles on the end mirrors surface. For the eigenstate $u_{\mathrm{A}}$, the field amplitude at the constant$z$ plane of the end mirror is $\mathcal{G} u_{\mathrm{A}}$. For its image eigenstate $u_{B} \equiv \mathcal{F} u_{\mathrm{A}}^{*}$, we have

$$
\mathcal{G} u_{\mathrm{B}}=\mathcal{G}\left(\mathcal{F} u_{\mathrm{A}}^{*}\right)=\left[\mathcal{G}^{*} \mathcal{F}^{-1} u_{\mathrm{A}}\right]^{*}=\left[i e^{i k L} R_{\left[\vec{r}^{2} /(2 L)\right]} \mathcal{G} u_{\mathrm{A}}\right]^{*},
$$

which does have the same intensity profile [see Eq. (3.4)].

For the round-trip propagator $\mathcal{L}^{2}$, using Eqs. (3.13) and (3.8), we have

$$
\left[\mathcal{L}_{\left[h_{\mathrm{A}}\right]}^{2}\right]^{*}=e^{4 i k L} \mathcal{F}^{-1} \mathcal{L}_{\left[h_{\mathrm{B}}\right]}^{2} \mathcal{F}
$$

so we have the same duality correspondence (3.18) between eigenstates of the full propagator, with the mapping between eigenvalues given by

$$
\eta_{\mathrm{B}}=e^{-4 i k L} \eta_{\mathrm{A}}^{*}
$$

\section{APPLICATION OF THE DUALITY RELATION USING MESA BEAMS AND MEXICAN-HAT CAVITIES}

The Mesa beams were constructed to have flat-topped intensity profiles at the cavity mirrors with rapid falloff near mirror rims, in order to achieve lower thermal noises $[5,6,13]$. There are two versions of Mesa beams with the same intensity profile, the nearly flat and the nearly concentric. Cavities that support them (Mexican-hat cavities) are related by the duality relation, as realized by Savov and Vyatchanin [14], during their 
study of radiation-pressure-induced tilt instabilities. In this section, we shall explicitly construct these two fundamental modes and study their relations at the center of the cavity, and at the cavity mirrors. We will also discuss analytical features of the two modes that have not been obtained before. We will also give an example of how the calculation of the tilt instability can be dramatically simplified for nearly concentric Mexican-hat cavities, using the duality relation, based on results already obtained for the nearly flat configuration.

\section{A. Construction of Mesa Beams in Cartesian Coordinate System}

Nearly flat Mesa beams are constructed by coherently superimposing minimal Gaussians, namely Gaussian modes with the smallest possible spot size at the cavity mirrors, $\sigma_{\min }=\sqrt{L /(2 k)}$, whose axes are parallel to the cavity axis and lie within a cylinder centered at the cavity axis. At the middle of the cavity, the axes intercept with the constant- $z$ plane in a disk $\mathcal{D}$, with radius $p$. It is evident that such a construction will give a rather flat intensity profile in the central region of the end mirror with radius $\sim p$; beyond this radius, the intensity profile falls off as a Gaussian with decay length $\sigma_{\min }[\underline{5}, 6]$.

The complex amplitude of the nearly flat Mesa beam (fundamental mode of the corresponding cavity) at the center of the cavity is of the form

$$
v_{\mathrm{f}}(\vec{r})=\int_{\vec{r}_{0} \in \mathcal{D}} d^{2} \vec{r}_{0}\left(\frac{1}{\sqrt{2 \pi} \sigma}\right)^{2} e^{-\frac{\left(\vec{r}-\vec{r}_{0}\right)^{2}}{2 \sigma^{2}}}
$$

Here $\sigma$ is the waist size, which we leave general (rather than setting $\left.\sigma=\sigma_{\min }\right)$ for the moment. The duality image of $v_{\mathrm{f}}$ is

$$
\begin{aligned}
v_{\mathrm{c}}(\vec{r}) & =\left[\mathcal{F} v_{\mathrm{f}}^{*}\right](\vec{r})=\int_{\vec{r}_{0} \in \mathcal{D}} d^{2} \vec{r}_{0} e^{\frac{2 i k \vec{r} \cdot \vec{r}_{0}}{L}} \mathcal{F}\left[\left(\frac{1}{\sqrt{2 \pi} \sigma}\right)^{2} e^{-\frac{\vec{r}^{2}}{2 \sigma^{2}}}\right] \\
& =\int_{\vec{r}_{0} \in \mathcal{D}} d^{2} \vec{r}_{0} e^{\frac{2 i k \vec{r} \cdot \vec{r}_{0}}{L}}\left[\left(\frac{1}{\sqrt{2 \pi} \sigma_{*}}\right)^{2} e^{-\frac{\vec{r}^{2}}{2 \sigma_{*}^{2}}}\right]
\end{aligned}
$$

with

$$
\sigma \sigma_{*}=\frac{L}{2 k}=\sigma_{\min }^{2}
$$

When going from Eq. ( $\underline{4.1)}$ to Eq. ( $\underline{4.2})$, the Fourier transform has been completed by two steps. First, the spatial translation by $\vec{r}_{0}$ is replaced by the phase factor of $e^{\frac{2 i k \vec{r} \vec{r}_{0}}{L}}$, which represents a tilt of the propagation axis by an angle of $2 \vec{r}_{0} / L$. Second, the $\sigma$-Gaussians turn into $\sigma_{*}$-Gaussians. [This correspondence between Gaussians in fact reflects the duality between pairs of spherical cavities.] As a consequence, $v_{\mathrm{c}}$ represents the superposition of Gaussians with symmetry axes going through the cavity center, but with tilt angles distributed uniformly in a disk with radius $2 p / L$ - exactly the construction of a nearly concentric Mesa beam. In particular, Eq. (4.3) tells us that the minimal Gaussian would have turned into itself in this process. Hence we have shown explicitly the correspondence between the nearly flat and nearly concentric Mesa beams (the fundamental modes of the corresponding cavities).

\section{B. Profiles of Mesa Beams and Mirror Shapes}

In order to study Mesa beams in more detail, we adopt the cylindrical polar coordinate system $(r, \phi)$; the cylindrical symmetry of these beams will make the complex amplitude only depend on $r$. Equations (4.1) and (4.2), written in the polar coordinate system, become

$$
v_{\mathrm{f}}^{\text {waist }}(r, \phi)=\frac{1}{\pi w_{0}^{2}} \int_{0}^{p} r_{0} \mathrm{~d} r_{0} \int_{0}^{2 \pi} \mathrm{d} \phi_{0} e^{-\frac{r^{2}-2 r_{0} r \cos \left(\phi-\phi_{0}\right)+r_{0}^{2}}{w_{0}^{2}}},
$$

$$
v_{c}^{\text {waist }}(r, \phi)=\frac{1}{\pi w_{0}^{2}} \int_{0}^{p} r_{0} \mathrm{~d} r_{0} \int_{0}^{2 \pi} \mathrm{d} \phi_{0} e^{-\frac{r^{2}+2 i r_{0} r \cos \left(\phi-\phi_{0}\right)}{w_{0}^{2}}}
$$

Here $w_{0}=\sqrt{L / k}=\sqrt{2} \sigma_{\min }$ and $L$ is the total length of the cavity. Carrying out the angular integrations analytically, we get

$$
\begin{aligned}
& v_{\mathrm{f}}^{\text {waist }}(r)=\int_{0}^{p / w_{0}} 2 x_{0} e^{-\left(x^{2}+x_{0}^{2}\right)} I_{0}\left(2 x x_{0}\right) \mathrm{d} x_{0}, \\
& v_{\mathrm{c}}^{\text {waist }}(r)=\frac{1}{x} e^{-x^{2}} J_{1}\left(2 x p / w_{0}\right),
\end{aligned}
$$

where $x \equiv r / w_{0}$, and $I_{0}$ is the modified Bessel function of the first kind. Examples of normalized power distributions of nearly flat and nearly concentric Mesa beams are plotted in the upper panels of Fig. 3. In these plots, we take $p=4 w_{0}$, which corresponds to the configuration proposed for advanced LIGO (for reasons that will be explained in Subsection 4.C).

Let us analyze these amplitude distributions in more detail, in the case of $p \gg w_{0}$, i.e., when we translate the minimal Gaussians by a distance substantially greater than their waist size. For the nearly flat configuration, we can easily see from Eq. ( $\underline{4.1})$ that, when $(p-r) / w_{0} \gg 1$, the field distribution can be approximated as
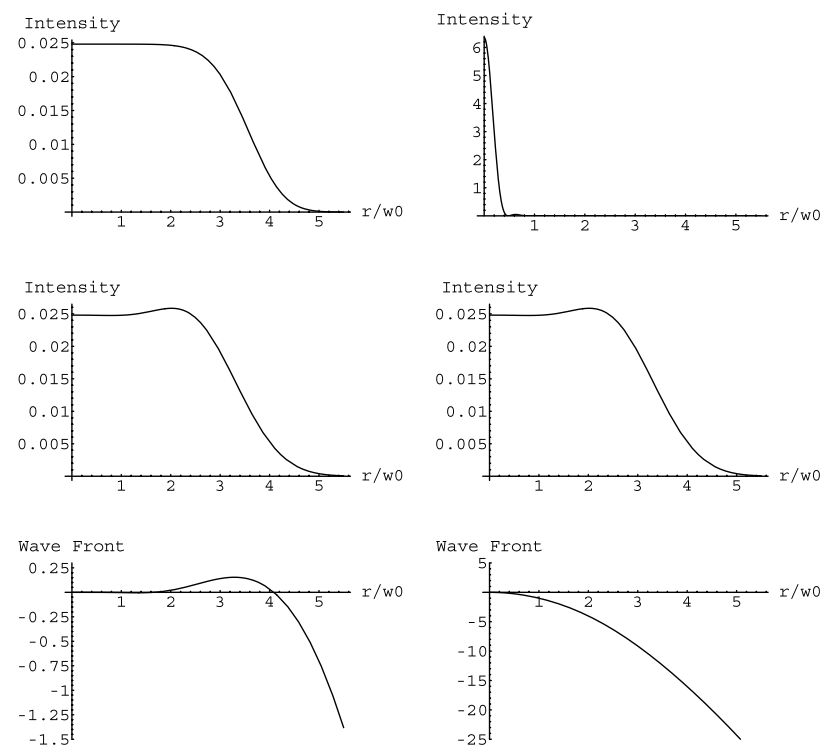

Fig. 3. Comparison between nearly flat (left panels) and nearly concentric (right panels) Mesa beams. Upper panels: normalized intensity profiles at the center of the cavity. Middle panels: normalized intensity profiles at mirror surfaces. Lower panels: phase fronts at the position of the mirrors. 


$$
v_{\mathrm{f}}(r \ll p) \approx \int_{\vec{r}_{0} \in \mathbf{R}^{2}} \mathrm{~d}^{2} \vec{r}_{0}\left(\frac{1}{\sqrt{2 \pi} \sigma}\right)^{2} e^{-\frac{\left(\vec{r}-\vec{r}_{0}\right)^{2}}{2 \sigma^{2}}}=1 .
$$

On the other hand, if $r$ is much larger than $w_{0}$ [since $p \gg w_{0}$, this region overlaps with the previous one], we can apply the asymptotic expansion of $I_{0}$

$$
I_{0}(z)=\frac{1}{\sqrt{2 \pi z}} e^{z}
$$

on Eq. (4.6), and obtain

$$
\begin{aligned}
v_{\mathrm{f}}^{\text {waist }}\left(r \gg w_{0}\right) & \approx \frac{1}{\sqrt{\pi}} \int_{0}^{p / w_{0}} \sqrt{\frac{x_{0}}{x}} e^{-\left(x_{0}-x\right)^{2}} \mathrm{~d} x_{0} \\
& \approx \frac{1}{\sqrt{\pi}} \int_{-x}^{p / w_{0}-x}\left(1+\frac{y}{2 x}\right) e^{-y^{2}} \mathrm{~d} y \\
& \approx\left[\frac{1}{\sqrt{\pi}} \int_{-\infty}^{p / w_{0}-x} e^{-y^{2}} \mathrm{~d} y\right]-\frac{1}{4 \sqrt{\pi} x} e^{-\left(p / w_{0}-x\right)^{2}},
\end{aligned}
$$

where we have defined $x \equiv r / w_{0}$. From Eq. (4.10), we note that when $w_{0} \ll r \ll p$, we recover the result of $v_{f}^{\text {waist }} \approx 1$; when $r$ gets close to $p$, the amplitude will drop, similar to the tail of an error function. Qualitatively, we could write $w_{\text {f-Mesa }}(p) \sim p$. In the ultimate limit of $p / w_{0} \rightarrow+\infty$, we have

$$
v_{\mathrm{f}}^{\text {waist }}(r)=1, \quad p / w_{0} \rightarrow+\infty .
$$

The concentric configuration, on the other hand, has a completely different field distribution. According to the analytic expression (4.7), the amplitude must be distributed within a radius of $x \sim w_{0} / p \ll 1$, or $r \sim w_{0}^{2} / p$, which is much smaller than the waist size of the minimal Gaussian. In this case, we could also qualitatively write $w_{\text {c-Mesa }}(p) \sim w_{0}^{2} / p$. In the limit of $p \rightarrow \infty$, we use

$$
\frac{J_{1}(a x)}{x} \rightarrow \delta(x), \quad a \rightarrow+\infty
$$

and have

$$
v_{\mathrm{c}}^{\text {waist }}(r)=\delta(x), \quad p / w_{0} \rightarrow+\infty \text {. }
$$

The fact that

$$
w_{\mathrm{f}-\mathrm{Mesa}}(p) \cdot w_{c \text {-Mesa }}(p) \sim w_{0}^{2}
$$

clearly reflects the Fourier-transform relation between two Mesa beams with the same $p$.

Now, let us turn to field distributions at the cavity mirrors. Applying the propagator between parallel planes in the polar coordinate systems [Eq. (3.5)],

$$
G\left(r^{\prime}, \phi^{\prime} ; r, \phi\right)=\frac{i k}{\pi L} e^{-i k L / 2} e^{-i k\left[r^{2}+r^{2}-2 r r^{\prime} \cos \left(\phi^{\prime}-\phi\right)\right] / L},
$$

we obtain the fields

$$
v_{\mathrm{f}}^{\text {end }}\left(r^{\prime}, \phi^{\prime}\right)=\int_{0}^{p} r_{0} \mathrm{~d} r_{0} \int_{0}^{2 \pi} \mathrm{d} \phi_{0} e^{-\left[\frac{1+i}{2}\right]\left[\frac{r^{\prime 2}-2 r_{0} r^{\prime} \cos \left(\phi^{\prime}-\phi_{0}\right)+r_{0}^{2}}{w_{0}^{2}}\right]},
$$

$v_{\mathrm{c}}^{\text {end }}\left(r^{\prime}, \phi^{\prime}\right)=\int_{0}^{p} r_{0} \mathrm{~d} r_{0} \int_{0}^{2 \pi} \mathrm{d} \phi_{0} e^{-\left[\frac{1+i}{2}\right]\left[\frac{r^{\prime 2}+2 i r_{0} r^{\prime} \cos \left(\phi^{\prime}-\phi_{0}\right)-i r_{0}^{2}}{w_{0}^{2}}\right]}$,

at distance $L / 2$ from the waist. Comparing Eqs. (4.16) and (4.17), we have

$$
\left[v_{\mathrm{f}}^{\text {end }}(\vec{r})\right]^{*}=e^{i k \vec{r}^{2} / L} v_{\mathrm{c}}^{\text {end }}(\vec{r})
$$

It is then obvious that the two beams have the same intensity profiles on the cavity mirrors:

$$
\left|v_{\mathrm{f}}^{\text {end }}(\vec{r})\right|=\left|v_{\mathrm{c}}^{\text {end }}(\vec{r})\right| \text {. }
$$

(An approximate formula for the end-mirror intensity profile was given in the appendix of [6].) We plot these intensity profiles at the mirror surfaces in the middle panels of Fig. 3 .

Let us now determine mirror shapes by imposing that the optical phase is constant (which we take as 0 for simplicity) on each mirror surface. We have

$$
\begin{aligned}
& v_{\mathrm{f}}^{\text {end }}(\vec{r}) e^{i k h_{\mathrm{f}}(\vec{r})}=\left|v_{\mathrm{f}}^{\text {end }}(\vec{r})\right|, \\
& v_{\mathrm{c}}^{\text {end }}(\vec{r}) e^{i k h_{\mathrm{c}}(\vec{r})}=\left|v_{\mathrm{c}}^{\text {end }}(\vec{r})\right| .
\end{aligned}
$$

Taking the complex conjugate of Eq. (4.20), and combining with Eq. (․․21), using Eqs. (‥18) and (‥19), we have

$$
h_{\mathrm{f}}(\vec{r})=\frac{\vec{r}^{2}}{L}-h_{\mathrm{c}}(\vec{r})
$$

which is the duality relation between mirror surfaces. In the lower panels of Fig. 4, we plot the shapes of mirror surfaces; again, we assume $p=4 w_{0}$.

\section{Applications of Mesa Beams to Advanced LIGO}

In order to achieve lower thermal noise in the test masses, the intensity profiles at the mirrors must be as flat as possible. In the case of infinite mirrors, the choice is to use cavities with flat or concentric spherical mirrors, whose eigenmodes have uniform (absolutely flat) profile distribution. However, the mirrors must have finite sizes (e.g., as limited by the size of the beam tube), and the intensity profiles must be confined to a very large extent within the rims of the mirrors, in order to decrease the diffraction loss upon each reflection. In advanced LIGO, a power loss below $10 \mathrm{ppm}$ is required [6].
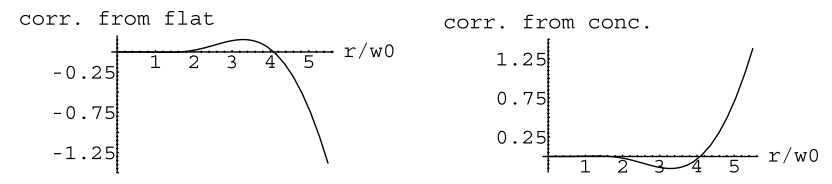

Fig. 4. Flat Mesa beam wave front (left panel) with respect to a flat surface and concentric Mesa beam wave front (right panel) with respect to a concentric surface, as analytically computed. 
For this reason, we are forced to deviate from flat or concentric configurations - to such an extent that the diffraction loss is within the requirement. When only spherical mirrors are used, if on the one hand we decrease the radius of curvature from $+\infty$ (flat), and on the other hand increase the radius of curvature from $L / 2$ (concentric), the dual configurations, with

$$
1 /\left(2 R_{1}\right)+1 /\left(2 R_{2}\right)=1 / L
$$

will have the same intensity profiles at the end mirrors, thus the same diffraction loss and thermal noise. For example, $R_{1}=54 \mathrm{~km}$ and $R_{2}=2.077 \mathrm{~km}$ both give exactly the loss specification, while $R_{1}$ is the current baseline design. However, spherical cavities are not optimal in terms of their thermal noise: (the two types of) Mesa beams, whose intensity profiles are flatter given the same loss specification, turn out to provide much lower thermal noises $[6,13]$. For these beams, the larger the parameter $p$, the lower the thermal noises, but the higher the diffraction loss. The loss specification of advanced LIGO corresponds to $p=4 w_{0}$ [6], which is the case we study in Fig. 3.

While having the same diffraction losses and thermal noises, dual configurations do differ significantly in a very important aspect-their eigenspectra are different. Thus, any problem using modal analysis of optical cavities will reveal these differences and probably the duality relation if nearly flat and nearly concentric configurations are compared.

One such problem is the radiation-pressure-induced tilt instability: as the mirrors tilt, the beam inside the cavity walks away from the center of the mirrors, producing a torque, which in some cases can drive more tilt in the same direction, and become destabilizing (see Fig. 5). As shown by Sigg [15], while for all cavities there is always one tilt mode in which the radiation-pressure-induced torque is destabilizing, the instability is much weaker in nearly concentric configurations than in nearly flat ones. The reason is that while in the two cases the intensity profiles are identical, the optical axis of the beam walks away by a much smaller distance in the concentric case, given the same amount of tilt in the unstable mode (see Fig. 5). According to Sigg's calculation for spherical

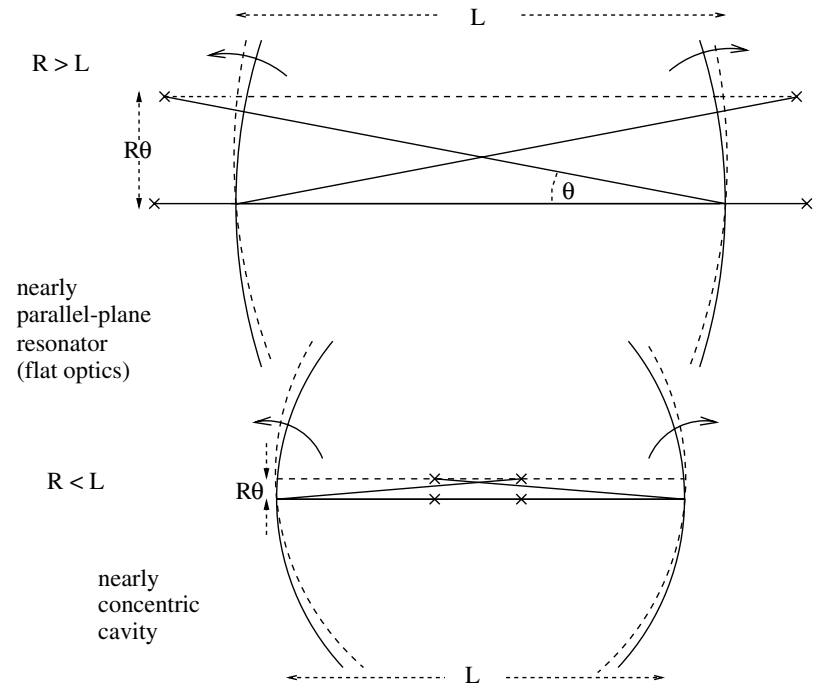

Fig. 5. Comparison of tilt instability of nearly flat and nearly concentric symmetric optical cavities. For more details, see [14,15]. mirrors, the tilt instability for a nearly flat configuration with Advanced-LIGO power ( $\sim 1$ MW circulating in the cavity) can be too strong to handle for the angular control system. For this reason, we would prefer nearly concentric cavities.

For general, nonspherical cavities, a perturbative prescription for calculating the tilt instability has been formulated by Savov and Vyatchanin [14], in which the tilt instability growth time is expressed in terms of eigenvalues and intensity profiles of the cavities' spatial eigenmodes (Eqs. 2.13, 2.14, and 4.8 of [14]). Savov and Vyatchanin applied their prescription to both nearly flat and nearly concentric Mexican-hat cavities; in particular, they had to solve the eigenvalue problem for the nearly concentric cavities in order to obtain the eigenvalues and intensity profiles. Savov discovered the duality relation in this process. Had the duality relation been known, one could have taken the eigenvalues and intensity profiles of nearly flat Mexican-hat cavities, available from previous works, applied the duality transformation, and obtained the tilt instability for nearly concentric Mexican-hat cavities without having to solve the eigenvalue problem again (see Section VI of [14]).

Finally, let us make a qualitative comment on the numerical magnitudes of tilt instabilities in the various configurations considered. Numerically, according to Savov and Vyatchanin [14], we have

$$
\begin{gathered}
\text { nearly flat Mexican hat (MH) }\left(p=4 w_{0}\right) \\
\downarrow \\
\text { nearly flat spherical }(R=54 \mathrm{~km}) \\
\downarrow \\
\text { nearly concentric spherical }(R=2.077 \mathrm{~km}) \\
\downarrow \\
\text { nearly concentric } \operatorname{MH}\left(p=4 w_{0}\right)
\end{gathered}
$$

with configurations less and less unstable from top to bottom. Interestingly, this sequence of decreasing instability is consistent qualitatively with the corresponding mirror shapes: with the same amount of diffraction loss, the flat MH does appear more flat than the nearly flat spherical mirrors, while the nearly concentric Mexican-hat mirror does appear closer to concentric than the nearly concentric spherical mirror.

\section{CONCLUSION}

In this paper, we provided two different analytic proofs for a duality relation between symmetric cavities with mirror height $h(\vec{r})$ measured with respect to a flat surface and those with mirror height $-h(\vec{r})$ measured with respect to a concentric spherical surface (valid within the paraxial approximation): the corresponding eigenmodes have the same intensity profile at the mirrors; their amplitude distribution at the center of the cavity is related via Fourier transform, while their eigenvalues are related by complex conjugation (see Table 1). These two proofs are based on the mirrorto-mirror propagator and the center-to-center propagator, respectively.

We illustrated this duality relation with the two types of Mesa beams proposed for advanced LIGO. In particular, we showed explicitly that these beams are related to each other by a Fourier transform at the center of the cavities, and that they have the same intensity profiles at the end of the cavities. We also related the mirror shapes of the Mexican-hat cavities that support these two modes by the duality relation. The duality relation could have allowed us to avoid solving the 
eigenequations once more for the nearly concentric Mexicanhat cavities, and used instead results already available for nearly flat Mexican-hat cavities.

In addition, this duality relation can also be applied to more general optical cavities, which interpolate between nearly flat and nearly concentric ones [17].

This duality relation provides a quite general tool for designing nonstandard optical cavities and for studying the performances of dual configurations. Several physical effects such as coupling of optical cavities with particles [25], coupling of mechanical and optical degrees of freedom in optical resonators [26], and cavity misalignment sensitivity, depend on the optical cavity modes structure in terms of beam geometry and eigenvalues. Therefore this unique mapping might be useful not only for studying practical issues related to advanced ground-based gravitational wave interferometers such as the parametric instability [27], but in a variety of other applications which could benefit from using Fabry-Perot cavities with nonspherical mirrors, including precision metrology and atomic physics. Ultrastable optical cavities have become a standard tool for stabilizing laser systems needed, for example, for high-resolution spectroscopy and optical clocks. Current cavities [28] are mostly limited by the mirrors' thermal noise and this may be reduced, inter alia, by using nonspherical mirrors supporting non-Gaussian beam. Particle manipulation in optical resonators is based on electric dipole interaction with the laser fields (optical dipole traps [29]) and the potential energy of the induced dipole force is related to the intensity distribution of the laser beam. Nonspherical mirrors could be employed for the optimization of the geometry and depth of the optical potential.

\section{APPENDIX A: DUALITY RELATION FOR NONIDENTICAL MIRRORS}

In this appendix we will study the duality relation in the case of nonidentical mirror shapes, but still symmetric under a $180^{\circ}$ rotation around the cavity axis. Now the field distributions of eigenstates over the two mirror surfaces are not identical and we have to study the eigenvalue problem associated with the round-trip propagator. Nevertheless, we can still use the propagators (2.5) and (2.7) to build a system of integral equations relating field distributions $v_{1}\left(\vec{r}_{1}\right)$ and $v_{2}\left(\vec{r}_{2}\right)$ over the two mirror surfaces. [All through this appendix, we use the subscripts 1 and 2 to refer to quantities associated with mirrors 1 and 2, respectively.] If the mirrors deviate from parallel planes by $h_{1,2}(\vec{r})$, we have

$$
\begin{aligned}
& \gamma_{1} v_{1}\left(\vec{r}_{1}\right)=\int_{S_{2}} \mathrm{~d}^{2} \vec{r}_{2} \mathcal{K}_{12}\left(\vec{r}_{1}, \vec{r}_{2}\right) v_{2}\left(\vec{r}_{2}\right), \\
& \gamma_{2} v_{2}\left(\vec{r}_{2}\right)=\int_{S_{1}} \mathrm{~d}^{2} \vec{r}_{1} \mathcal{K}_{21}\left(\vec{r}_{2}, \vec{r}_{1}\right) v_{1}\left(\vec{r}_{1}\right),
\end{aligned}
$$

where $\gamma_{1,2}$ are the "eigenvalues" and

$$
\begin{aligned}
& \mathcal{K}_{12}\left(\vec{r}_{1}, \vec{r}_{2}\right)=\frac{i k e^{-i k L}}{2 \pi L} e^{i k h_{1}\left(\vec{r}_{1}\right)-\frac{i k}{2 L}\left|\vec{r}_{1}-\vec{r}_{2}\right|^{2}+i k h_{2}\left(\vec{r}_{2}\right)}, \\
& \mathcal{K}_{21}\left(\vec{r}_{2}, \vec{r}_{1}\right)=\frac{i k e^{-i k L}}{2 \pi L} e^{i k h_{2}\left(\vec{r}_{2}\right)-\frac{i k}{2 L}\left|\vec{r}_{2}-\vec{r}_{1}\right|^{2}+i k h_{1}\left(\vec{r}_{1}\right)}
\end{aligned}
$$

are the propagators from mirror 2 to mirror 1, and from mirror 1 to mirror 2, respectively. Equations (A1) and (A2) give the field at each mirror in terms of the reflected field at the other, but they can be combined to form the round-trip equation, which states that the field at each mirror must reproduce itself after one round-trip. In the following, we will add a subscript $f$ or $c$ to make a distinction between quantities related to the nearly flat or nearly concentric case.

$$
\begin{aligned}
& \eta_{f} v_{1 f}\left(\vec{r}_{1}\right)=\int_{S_{1}^{\prime}} \mathrm{d}^{2} \vec{r}_{1}^{\prime} \mathcal{K}_{1 f}^{h_{1} h_{2}}\left(\vec{r}_{1}, \vec{r}_{1}^{\prime}\right) v_{1 f}\left(\vec{r}_{1}^{\prime}\right), \\
& \eta_{f} v_{2 f}\left(\vec{r}_{2}\right)=\int_{S_{2}^{\prime}} \mathrm{d}^{2} \vec{r}_{2}^{\prime} \mathcal{K}_{2 f}^{h_{2} h_{1}}\left(\vec{r}_{2}, \vec{r}_{2}^{\prime}\right) v_{2 f}\left(\vec{r}_{2}^{\prime}\right),
\end{aligned}
$$

where the common eigenvalue $\eta_{f}$ is given by $\gamma_{1 f} \gamma_{2 f}$ and the round-trip propagators

$$
\begin{aligned}
& \mathcal{K}_{1 f}^{h_{1} h_{2}}\left(\vec{r}_{1}, \vec{r}_{1}^{\prime}\right)=\int_{S_{2}} \mathrm{~d}^{2} \vec{r}_{2} \mathcal{K}_{12 f}\left(\vec{r}_{1}, \vec{r}_{2}\right) \mathcal{K}_{21 f}\left(\vec{r}_{2}, \vec{r}_{1}\right), \\
& \mathcal{K}_{2 f}^{h_{2} h_{1}}\left(\vec{r}_{2}, \vec{r}_{2}^{\prime}\right)=(1 \leftrightarrow 2) \cdot \mathcal{K}_{1 f}^{h_{1} h_{2}}\left(\vec{r}_{1}, \vec{r}_{1}^{\prime}\right) .
\end{aligned}
$$

In the nearly concentric configuration, using kernels of the form (2.7) for the propagation from one mirror to the other and combining them as done for the nearly flat configuration, we obtain the following nearly concentric round-trip equation for the field distribution over the mirror 1 (similar formula for the mirror 2 with the substitution $1 \leftrightarrow 2$ ).

$$
\begin{aligned}
\eta_{c} v_{1 c}\left(\vec{r}_{1}\right)= & \int_{S_{1}^{\prime}} \mathrm{d}^{2} \vec{r}_{1}^{\prime} \mathcal{K}_{1 c}^{b_{1} b_{2}}\left(\vec{r}_{1}, \vec{r}_{1}^{\prime}\right) v_{1 c}\left(\vec{r}_{1}\right) \\
\mathcal{K}_{1 c}^{b_{1} b_{2}}\left(\vec{r}_{1}, \vec{r}_{1}^{\prime}\right)= & -\int_{S_{2}} \mathrm{~d}^{2} \vec{r}_{2} e^{-2 i k L}\left(\frac{k}{2 \pi L}\right)^{2} \\
& \cdot e^{\frac{i k}{2 L} \vec{r}_{1}+\left.\vec{r}_{2}\right|^{2}+\frac{i k}{2 L}\left|\vec{r}_{2}+\vec{r}_{1}\right|^{2}+i k b_{1}\left(\vec{r}_{1}\right)+i k b_{1}\left(\vec{r}_{1}\right)+2 i k b_{2}\left(\vec{r}_{2}\right)},
\end{aligned}
$$

where $b_{1,2}$ are the mirror deviations from concentric surfaces. Using the assumed symmetry properties of the mirrors, the propagators for the nearly flat and nearly concentric cavity fulfill this relation (the same is true for the mirror 2 with the substitution $1 \leftrightarrow 2$ )

$$
\begin{aligned}
\mathcal{K}_{1 c}^{-h_{1}-h_{2}}\left(\vec{r}_{1}, \vec{r}_{1}^{\prime}\right) & =e^{-4 i k L}\left[\mathcal{K}_{1 f}^{h_{1} h_{2}}\left(-\vec{r}_{1},-\vec{r}_{1}^{\prime}\right)\right]^{*} \\
& =e^{-4 i k L}\left[\mathcal{K}_{1 f}^{h_{1} h_{2}}\left(\vec{r}_{1}, \vec{r}_{1}^{\prime}\right)\right]^{*}
\end{aligned}
$$

Equation (A10), together with Eqs. (A7) and (A8), express a general duality relation for cavities with nonidentical mirrors: as long as the corresponding mirrors of two cavities $A$ and $B$ satisfy

$$
h_{\alpha A}(\vec{r})=\frac{\vec{r}^{2}}{L}-h_{\alpha B}(\vec{r}), \quad \alpha=1,2
$$

the eigenstates and eigenvalues of the two cavities will be related by

$$
v_{\alpha A}=v_{\alpha B}^{*}, \quad \eta_{A}=e^{-4 i k L} \eta_{B}^{*}, \quad \alpha=1,2 .
$$




\section{APPENDIX B: EIGENSTATES AND EIGENVALUES FOR CAVITIES WITH INFINITE MIRRORS}

When the mirrors are infinite, it is straightforward to check that two fundamental properties,

$$
\begin{gathered}
\int \mathrm{d}^{2} \vec{r}^{\prime} \mathcal{K}\left(\vec{r}, \vec{r}^{\prime}\right) \mathcal{K}^{*}\left(\vec{r}^{\prime}, \vec{r}^{\prime \prime}\right)=\delta\left(\vec{r}-\vec{r}^{\prime \prime}\right), \\
\mathcal{K}\left(\vec{r}, \vec{r}^{\prime}\right)=\mathcal{K}\left(\vec{r}^{\prime}, \vec{r}\right),
\end{gathered}
$$

are satisfied by both propagators $\mathcal{K}_{\mathrm{f}}^{h}$ and $\mathcal{K}_{\mathrm{c}}^{b}$; they can be rewritten into

$$
\mathcal{K} \mathcal{K}^{\dagger}=\mathbf{I}, \quad \mathcal{K}=\mathcal{K}^{T},
$$

where $\mathbf{I}$ is identity operator, $\mathcal{K}^{T}$ is the conjugate of $\mathcal{K}$, and $\mathcal{K}^{\dagger}$ its Hermitian conjugate. In simple terms, $\mathcal{K}$ is unitary and symmetric. It is well known that for unitary operators, all eigenvalues have modulus 1 , and that eigenvectors with different eigenvalues are orthogonal to each other.

Now suppose we have an eigenvector $v$, with eigenvalue $\gamma$, $\gamma \gamma^{*}=1$. By complex conjugating the eigenequation $\mathcal{K} v=\gamma v$, we obtain

$$
\mathcal{K}^{*} v^{*}=\gamma^{*} v^{*}=\gamma^{-1} v^{*}
$$

using Eqs. (B3), we have $\mathcal{K}^{*}=\mathcal{K}^{\dagger}=\mathcal{K}^{-1}$, and hence

$$
\mathcal{K}^{-1} v^{*}=\gamma^{*} v^{*} \Rightarrow \gamma v^{*}=\mathcal{K} v^{*}
$$

This means $v^{*}$ and $v$ are both eigenvectors with eigenvalue $\gamma$. We can then replace $v$ and $v^{*}$ by two real eigenvectors of the eigenvalue problem, $v+v^{*}$ and $\left(v-v^{*}\right) / i$. This corresponds to the physical fact that the optical phase of eigenstates must be constant on mirror surfaces.

\section{ACKNOWLEDGMENTS}

We thank K.S. Thorne and W. Kells for useful discussions. Research of P.S. and Y.C. was supported by the National Science Foundation under grant No. PHY-0099568. Research of Y.C. was also sponsored by the Alexander von Humboldt Foundation's Sofja Kovalevskaja Award (funded by the German Federal Ministry of Education and Research) and the David and Barbara Groce fund at the San Diego Foundation. Research of J.A. and E.D'A. was supported by National Science Foundation under grant No. PHY-0107417.

\section{REFERENCES AND NOTES}

1. LIGO http://www.ligo.caltech.edu.

2. Advanced LIGO https://www.advancedligo.mit.edu.

3. The name mesa beam was invented by P. Willems.

4. K. S. Thorne, LIGO-G000068-00-D (2000), available at http:// admdbsrv.ligo.caltech.edu/dcc/.

5. E. D'Ambrosio, R. O'Shaughnessy, S. Strigin, K. S. Thorne, and S. Vyatchanin, "Reducing thermoelastic noise in gravitationalwave interferometers by flattening the light beams," arXiv:grqc/0409075v1.

6. R. O'Shaughnessy, S. Strigin, and S. Vyatchanin, "The implications of Mexican-hat mirrors: calculations of thermoelastic noise and interferometer sensitivity to perturbation for Mexican-hat mirror proposal for advanced LIGO," arXiv:gr-qc/0409050v1.

7. E. D'Ambrosio, "Non-spherical mirrors to reduce thermoelastic noise in advanced gravity wave interferometers," Phys. Rev. D 67, 102004 (2003).

8. M. G. Tarallo, J. Miller, J. Agresti, E. D’Ambrosio, R. DeSalvo, D. Forest, B. Lagrange, J. M. Mackowski, C. Michel, J. L. Montorio, N. Morgado, L. Pinard, A. Remilleux, B. Simoni, and P. Willems, "Generation of a flat-top laser beam for gravitational-wave detectors by means of a non-spherical Fabry-Perot resonator," Appl. Opt. 46, 6648-6654 (2007).

9. M. Bondarescu, O. Kogan, and Y. Chen, "Optimal light beams and mirror shapes for future LIGO interferometers," Phys. Rev. D 78, 082002 (2008).

10. B. Mours, E. Tournefier, and J. Y. Vinet, "Thermal noise reduction in interferometric gravitational wave antennas: using high order TEM modes," Classical Quantum Gravity 23, 5777-5784 (2006).

11. J. Y. Vinet, "On special optical modes and thermal issues in advanced gravitational wave interferometric detectors," Living Rev. Relativity 12, 5 (2009).

12. V. Pierro, V. Galdi, G. Castaldi, I. M. Pinto, J. Agresti, and R. DeSalvo, "Perspectives on beam-shaping optimization for thermal-noise reduction in advanced gravitational-wave interferometric detectors: bounds, profiles, and critical parameters," Phys. Rev. D 76, 122003 (2007).

13. J. Agresti and R. DeSalvo, "Thermal noises calculations: Gaussian vs. Mesa beams," LIGO technical note, LIGO-T050269-00-R available at http://admdbsrv.ligo.caltech.edu/dcc/.

14. P. Savov and S. Vyatchanin, "Estimate of tilt instability of Mesa-beam and Gaussian-beam modes for advanced LIGO," Phys. Rev. D 74, 082002 (2006).

15. D. Sigg, "Angular instability in high power FP cavities," LIGO technical note, LIGO-T030120-00, (2003); available at http:// admdbsrv.ligo.caltech.edu/dcc/.

16. J. Sidles and D. Sigg, "Optical torques in suspended Fabry-Perot interferometers,” Phys. Lett. A 354, 167-172 (2006).

17. M. Bondarescu and K. S. Thorne, "A new family of light beams and mirror shapes for future LIGO interferometers," Phys. Rev. D 74, 082003 (2006).

18. V. Galdi, G. Castaldi, V. Pierro, I. M. Pinto, J. Agresti, E. DAmbrosio, and R. DeSalvo, "Analytic structure of a family of hyperboloidal beams of potential interest for advanced LIGO," Phys. Rev. D 73, 127101 (2006).

19. A. P. Lundgren, R. Bondarescu, D. Tsang, and M. Bondarescu, "Finite mirror effects in advanced interferometric gravitational wavedetectors,” Phys. Rev. D 77, 042003 (2008).

20. A. G. Fox and T. Li, "Resonant modes in a maser interferometer," Bell Syst. Tech. J. 40, 453-488 (1961).

21. J. P. Gordon and H. Kogelnik, "Equivalence relations among spherical mirror optical resonators," Bell Syst. Tech. J. 43, 2873-2886 (1964).

22. H. Kogelnik and T. Li, "Laser beams and resonators," Appl. Opt. 5, 1550-1567 (1966).

23. G. Herziger and H. Weber, "Equivalent optical resonators," Appl. Opt. 23, 1450-1452 (1984).

24. A. Siegman, Lasers (University Science Books, 1996), Ch. 19.

25. P. Domokos and H. Ritsch, "Mechanical effects of light in optical resonators,” J. Opt. Soc. Am. B 20, 1098-1130 (2003).

26. V. B. Braginsky, S. E. Strigin, and S. P. Vyatchanin, "Parametric oscillatory instability in Fabry-Perot interferometer," Phys. Lett A 287, 331-338 (2001).

27. S. Gras, D. G. Blair, and L. Ju, "Opto-acoustic interactions in gravitational wave detectors: comparing flat-top beams with Gaussian beams,” Phys. Rev. D 81, 042001 (2010).

28. Y. Y. Jiang, A. D. Ludlow, N. D. Lemke, R. W. Fox, J. A. Sherman, L. S. Ma, and C. W. Oates, "Making optical atomic clocks more stable with 10-16-level laser stabilization," Nat. Photon. 5, 158161 (2011).

29. R. Grimm, M. Weidenmüller, and Y. B. Ovchinnikov, "Optical dipole traps for neutral atoms,” Adv. Atomic Molec. Opt. Phys. 42, 95-170 (2000). 